\title{
Sunlight-variation-adaptive charge pump circuit with self-reconfiguration for small-scale solar energy harvesting
}

\author{
Eun-Sub Lee, Jun-Myung Choi, Young-Su Kim, Seok-Jin Ham, \\ Jeong-Heon Kim, Sang-Don Byeon, and Kyeong-Sik Min ${ }^{\text {a) }}$ \\ School of Electrical Engineering, Kookmin University \\ 861-1, Jeongneung-dong, Seongbuk-gu, Seoul 136-702, Korea \\ a)mks@kookmin.ac.kr
}

\begin{abstract}
In this paper, we propose a reconfigurable charge pump (RCP) circuit that could change its architecture according to sunlight variation. In strong sunlight, the RCP works by the parallel mode that can maximize the output current. In moderate sunlight, the RCP changes to the serial-parallel mode. When sunlight becomes weak, the RCP runs by the serial mode to keep its output voltage around the target voltage. Compared with the fixed-mode circuit that maintains the serial mode all the time regardless of sunlight variation, the RCP can generate 2.5 times larger output current and has power efficiency better by $24 \%$, in strong sunlight. The area penalty and overhead in power efficiency of the RCP are only $15.8 \%$ and $1.2 \%$.
\end{abstract}

Keywords: sunlight-variation-adaptive, reconfigurable charge pump, solar energy harvesting, solar battery charger

Classification: Integrated circuits

\section{References}

[1] T. Esram and P. L. Chapman, "Comparison of photovoltaic array maximum power point tracking techniques," IEEE Trans. Energy Convers., vol. 22, no. 2, pp. 439-448, June 2007.

[2] J. Ahmad, "A fractional open circuit voltage based maximum power point tracker for photovoltaic arrays," Int. Conf. Software Technology Engineering, vol. 1, pp. 247-250, 2010.

[3] E. S. Lee, C. M. Jung, H. M. Vo, and K. S. Min, "Compact and efficient maximum power point tracking circuit for portable solar battery charger," IEICE Electron. Express, vol. 8, no. 12, pp. 930-937, June 2011.

[4] I. Doms, P. Merken, C. Van Hoof, and R. P. Mertens, "Capacitive Power Management Circuit for Micropower Thermoelectric Generators With a $1.4 \mu \mathrm{A}$ Controller," IEEE J. Solid-State Circuits, vol. 44, no. 10, pp. 2824-2833, 2009.

[5] H. Shao, C. Y. Tsui, and W. H. Ki, "The design of a Micro Power Management System for Applications Using Photovoltaic Cells with the 
Maximum Output Power Control," IEEE Trans. Very Large Scale Integr. (VLSI) Syst., vol. 17, no. 8, pp. 1138-1142, Aug. 2009.

[6] H. Gao, P. Baltus, R. Mahmoudi, and A. V. Roermund, "2.4 GHz Energy Harvesting for Wireless Sensor Network," 2011 IEEE Topical Conference on WiSNet, pp. 57-60, 2011.

[7] Brief datasheet of LIR1220, EEMB Inc. [Online] http://eemb.com

[8] M. Chen and G. A. Rincon-Mora, "Accurate, compact, and power efficient Li-Ion battery charger circuit," IEEE Trans. Circuits Syst. II, vol. 53, no. 11, pp. 1180-1184, Nov. 2006.

[9] J. Buxton, "Li-Ion battery charging requires accurate voltage sensing," Analog Devices: Analog Dialogue, vol. 31, no. 2, 1997.

[10] P. Favrat, P. Deval, and M. J. Declercq, "A high-efficiency CMOS voltage doubler," IEEE J. Solid-State Circuits, vol. 33, no. 3, pp. 410-416, March 1998.

[11] J. F. Dickson, "On-chip high-voltage generation in MNOS integrated circuits using an improved voltage multiplier technique," IEEE J. SolidState Circuits, vol. 11, no. 3, pp. 374-378, June 1976.

[12] F. Su and W. H. Ki, "An integrated reconfigurable SC Power converter with hybrid gate control scheme for mobile display driver applications," IEEE Asian Solid-State Circuits Conference, pp. 169-172, 2008.

[13] F. Pen and T. Samaddar, Charge Pump Circuit Design, McGraw-Hill Companies, New York, 2006.

[14] P. E. Allen and D. R. Holberg, CMOS analog circuit design, 2nd edition, OXFORD, 2002.

\section{Introduction}

Recently many systems that can harvest energy from their environments such as wind, sun, vibration, temperature, etc are being studied $[1,2,3,4$, 5]. Among them, solar energy is the most popular energy source due to its ubiquitous availability, high power density, and that DC voltage and current can be directly obtained from sunlight [3]. Solar energy can be converted by inductor-based power circuit or capacitor-based charge pump. Because the charge pump that does not need any off-chip devices, it is regarded more suitable to small-scale solar harvesting systems with lower cost and simpler circuit. One of the best examples of small-scale harvesting systems for solar energy is Wireless Sensor Node (WSN) system that should be implemented with a tiny low-power chip driven by small-capacity and small-size battery $[6$, 7].

Figure 1 (a) shows a typical current-voltage relationship of solar cell array with the sunlight intensity varying. As the intensity increases, the solar array current $\left(\mathrm{I}_{\mathrm{SA}}\right)$ and voltage $\left(\mathrm{V}_{\mathrm{SA}}\right)$ become larger, too. For a given sunlight intensity, the maximum available $\mathrm{V}_{\mathrm{SA}}$ is obtained by the open-circuit condition. The open-circuit $\mathrm{V}_{\mathrm{SA}}$ is defined by the open-circuit voltage $\left(\mathrm{V}_{\mathrm{OC}}\right)$. Figure 1 (b) shows a relationship between solar power $\left(\mathrm{P}_{\mathrm{SA}}\right)$ and $\mathrm{V}_{\mathrm{SA}}$. Here $\mathrm{P}_{\mathrm{SA}}$ can be simply calculated with $\mathrm{V}_{\mathrm{SA}} \times \mathrm{I}_{\mathrm{SA}}$. In this figure, a maximum power point (MPP) varies according to its sunlight condition. In strong sunlight, the MPP is observed at the point, $\mathrm{S}$ in Figure 1 (b) that can generate 


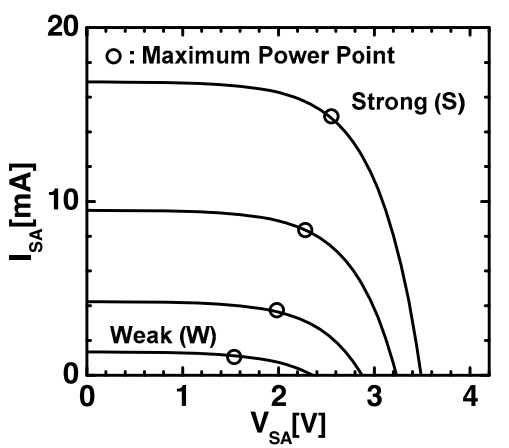

(a)

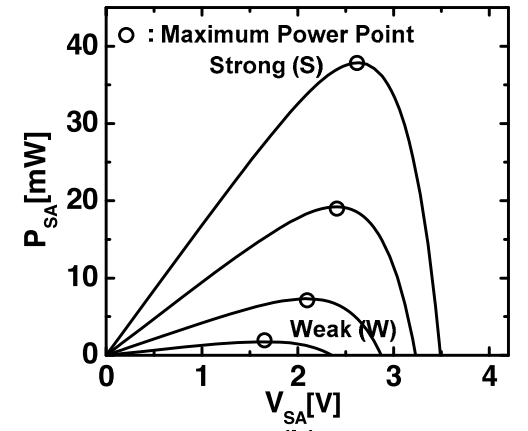

(b)

Fig. 1. (a) Solar array current and voltage relationship for different sunlight conditions (b) Solar power and voltage relationship for different sunlight conditions. Here, $\mathrm{W}$ and $\mathrm{S}$ represent the weak and strong sunlight conditions, respectively. The circle symbol represents the maximum power point at a given sunlight condition.

the largest power. When the intensity becomes as weak as the point, $\mathrm{W}$, the solar cell's power becomes much smaller, as shown in Figure 1 (b).

To design charge pump circuit for solar energy conversion, we need to decide the number of stages and the amount of output current of charge pump. Here, we assumed that the output voltage of charge pump should be $4.2 \mathrm{~V}$ that is required to charge Lithium batteries that are used popularly in most of mobile applications [8, 9]. In Figure 1 (a), if we look at the point, S, the maximum power point is around $2.5 \mathrm{~V}$ that is large enough to generate $4.2 \mathrm{~V}$ using only one-stage pump circuit. And, in Figure $1(\mathrm{a}), \mathrm{I}_{\mathrm{SA}}$ at the point, $\mathrm{S}$, is very large that means the solar cell array can deliver a large amount of solar current to the pump circuit. When the sunlight is as weak as the point, $\mathrm{W}$, the number of stages of charge pump should be larger than 2 because the $\mathrm{V}_{\mathrm{SA}}$ at this point is just around $1 / 3$ of the target $\mathrm{V}_{\text {OUT }}$. And, also, the amount of solar array current of the point, $\mathrm{W}$, is much smaller than the point, $\mathrm{S}$ that means the weak sunlight can generate smaller output current of charge pump circuit than the strong sunlight. The weak sunlight needs the larger number of stages and can generate small output current. On the contrary, the strong sunlight needs the smaller number of stages and is able to deliver larger output current to the load.

We can think of two typical architectures that are depicted by the parallel architecture and serial one, respectively, as shown in Figure 2 (a). Here $\mathrm{C}_{\mathrm{n}}$ is pumping capacitor and $\mathrm{V}_{\text {IN }}$ and $\mathrm{V}_{\text {OUT }}$ represent the input and output voltage of charge pump, respectively. In the parallel architecture, all the pumping capacitors are connected in parallel. On the contrary, in the serial architecture, the four pumping capacitors are connected in series. Figures 2 (b) and (c) compare the output current and power efficiency, respectively. Here the output voltage is fixed at $4.2 \mathrm{~V}$. In Figure $2(\mathrm{~b})$, when the boosting ratio of $\mathrm{V}_{\text {OUT }} / \mathrm{V}_{\text {IN }}$ is higher than 2, the parallel circuit generates the output current that is almost zero because the output voltage cannot reach the required volt- 


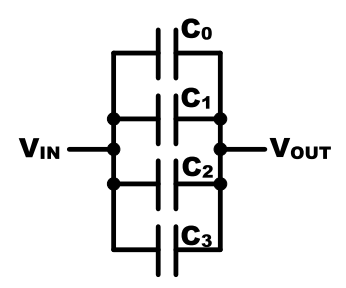

Parallel Architecture (Better for Strong Sunlight)

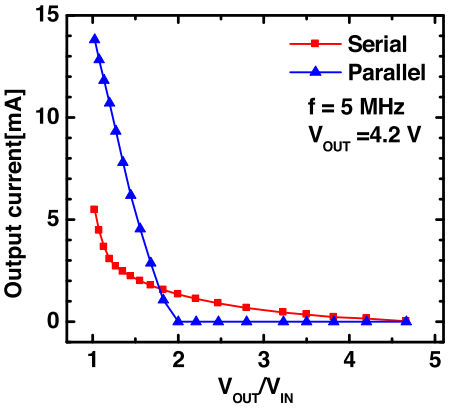

(b)

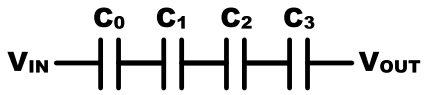

$\longleftrightarrow \quad$ Serial Architecture

(Better for Weak Sunlight)

(a)

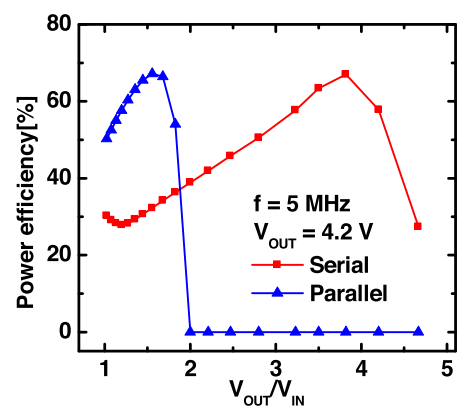

(c)
Fig. 2. (a) The parallel circuit architecture for strong sunlight and the serial circuit architecture for weak sunlight (b) the output current of charge pump with the boosting ratio of $\mathrm{V}_{\mathrm{OUT}} / \mathrm{V}_{\mathrm{IN}}$ varying (c) the power efficiency of charge pump with the boosting ratio of $\mathrm{V}_{\mathrm{OUT}} / \mathrm{V}_{\mathrm{IN}}$ varying.

age. As the boosting ratio of $\mathrm{V}_{\mathrm{OUT}} / \mathrm{V}_{\mathrm{IN}}$ decreases lower, the parallel circuit has larger output current than the serial due to that its effective pumping capacitance is much larger than the serial circuit. For the power efficiency, the serial circuit is better when the boosting ratio is high. The parallel circuit becomes better as the boosting ratio decreases lower, as shown in Figure 2 (c). Hence, from considering both Figures 1 and 2, the parallel architecture is more suitable to the strong sunlight. Similarly, the serial architecture can be regarded better for the weak sunlight.

\section{Reconfigurable charge pump circuit}

A conceptual schematic of Reconfigurable Charge Pump (RCP) circuit is shown in Figure $3(\mathrm{a})$, where $C_{n}$ is the pumping capacitor and $S_{n}$ is the control switch for reconfiguring charge pump's architecture. $\mathrm{V}_{\text {IN }}$ and $\mathrm{V}_{\text {OUT }}$ represent the input and output voltage of charge pump, respectively. The control switches in Figure 3 (a) can change to Parallel Mode (PM) that is shown in Figure $3(\mathrm{~b})$, when the sunlight is strong. In PM, $\mathrm{S}_{1}, \mathrm{~S}_{4}$, and $\mathrm{S}_{7}$ are off and the others are on thus all the pumping capacitors run in parallel to maximize the output current but the boosting ratio is minimized. When the sunlight becomes moderate, the charge pump starts to reconfigure its architecture by Serial-Parallel Mode (SPM). The control switches in SPM are shown in Figure 3 (c). In SPM, $\mathrm{C}_{0}$ and $\mathrm{C}_{1}$ are in series and $\mathrm{C}_{2}$ and $\mathrm{C}_{3}$ are 


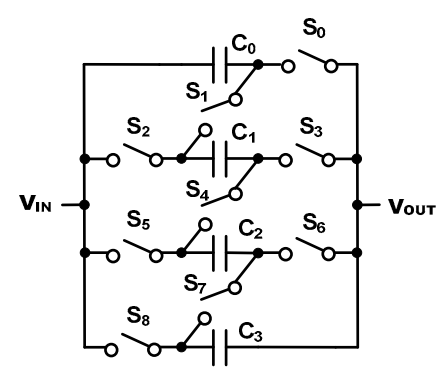

(a)

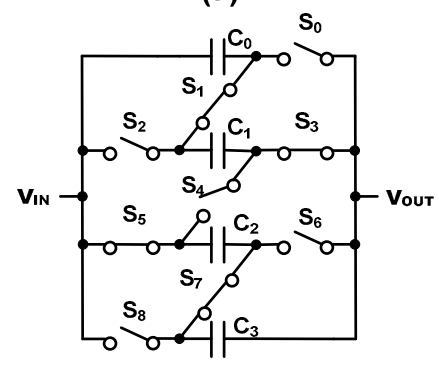

(c)

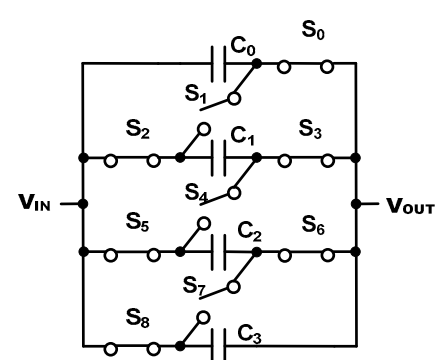

(b)

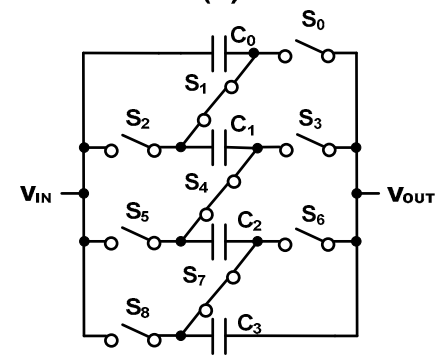

(d)

Fig. 3. (a) The conceptual schematic of Reconfigurable Charge Pump (RCP) circuit with control switches (b) Parallel Mode (PM) configuration (c) SerialParallel Mode (SPM) configuration (d) Serial Mode (SM) configuration.

in series but $\mathrm{C}_{0}-\mathrm{C}_{1}$ and $\mathrm{C}_{2}-\mathrm{C}_{3}$ are in parallel. Compared with PM, SPM can boost the output voltage higher than PM but delivers smaller output current to the load. Serial Mode (SM) is shown in Figure $3(\mathrm{~d})$. In this figure, $\mathrm{V}_{\mathrm{IN}}$ is transferred to $\mathrm{C}_{0}$ and the output voltage is boosted through $\mathrm{C}_{0}, \mathrm{C}_{1}, \mathrm{C}_{2}$, and $\mathrm{C}_{3}$ that are connected in series. In SM, because all the pumping capacitors are connected in series, the output voltage can be maximized but the output current is the smallest among three modes of PM, SPM, and SM.

The circuit implementation of Figure 3 (a) is shown in Figure 4 (a) that is composed of four CP cells that are controlled by the nine switches. Here $\mathrm{CP}$ cell represents one-stage charge pump that is shown in Figure 4 (b) and is based on the voltage doubler circuit [10] which has smaller voltage loss than the Dickson charge pump [11]. In Figure 4 (a), $\mathrm{V}_{\text {IN }}$ is the input voltage that comes from the solar cell array. $\mathrm{V}_{\text {OUT }}$ is the output voltage of RCP that goes into the load. The switches from $\mathrm{S}_{0}$ to $\mathrm{S}_{8}$ are used to reconfigure the mode according to sunlight variation. Each CP cell is controlled by two-phase clock signals that are CK and CKB. In Figure 4 (b), $\mathrm{M}_{0}$ and $\mathrm{M}_{2}$ are connected to $\mathrm{V}_{\mathrm{IN}}$ and $\mathrm{M}_{1}$ and $\mathrm{M}_{3}$ are to $\mathrm{V}_{1} \cdot \mathrm{C}_{0}$ and $\mathrm{C}_{1}$ are the pumping capacitors. When CK is high and CKB is low, $\mathrm{M}_{0}$ is on and $\mathrm{M}_{1}$ is off. At this time, $\mathrm{M}_{2}$ is off and $\mathrm{M}_{3}$ is on. In Figure 4 (b), the body of $\mathrm{M}_{\mathrm{SO}}$ is connected to $\mathrm{V}_{\text {OUT }}$. Similarly, the bodies of $\mathrm{M}_{\mathrm{S} 1}$ and $\mathrm{M}_{\mathrm{S} 2}$ are connected to $\mathrm{V}_{1}$ and $\mathrm{V}_{2}$, respectively. The bodies of $\mathrm{M}_{\mathrm{S} 0}, \mathrm{M}_{\mathrm{S} 1}$, and $\mathrm{M}_{\mathrm{S} 2}$ are biased not to turn on their body diodes during the operation. The gates of $\mathrm{M}_{\mathrm{S} 0}, \mathrm{M}_{\mathrm{S} 1}$, and $\mathrm{M}_{\mathrm{S} 2}$ are controlled by the switch controller circuit that is shown in Figure $4(\mathrm{c}) . \mathrm{M}_{\mathrm{SN}}$ that represents the $\mathrm{N}^{\text {th }}$ control switch such as $\mathrm{M}_{\mathrm{S} 0}$ is controlled by $\mathrm{M}_{\mathrm{P}}$ and $\mathrm{M}_{\mathrm{N}}$. When $\mathrm{M}_{\mathrm{P}}$ 


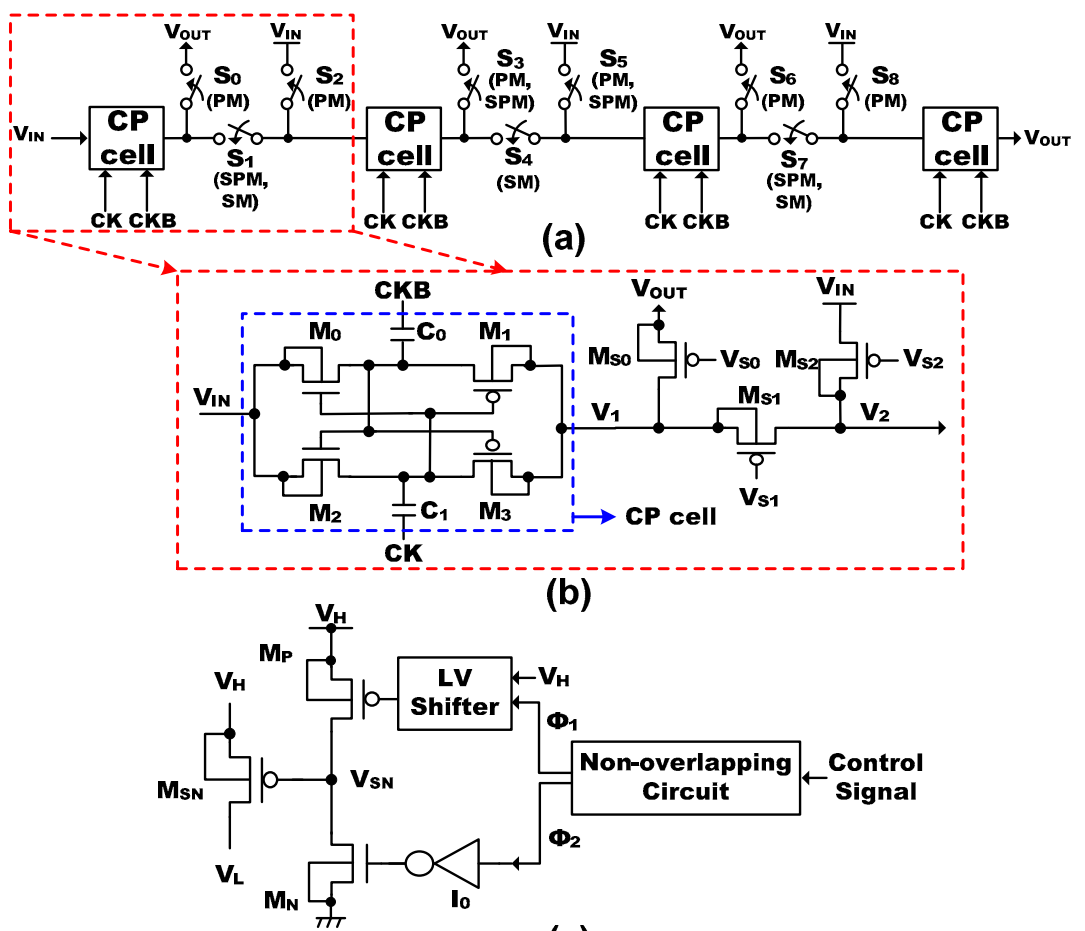

(c)

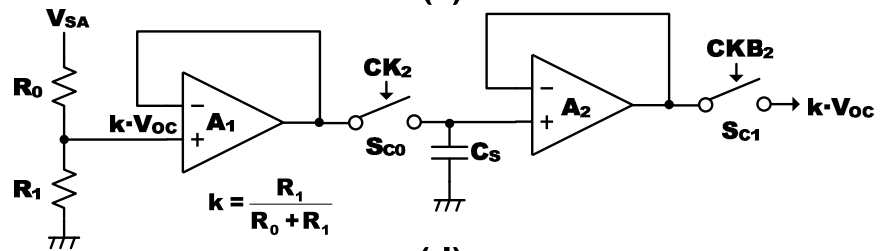

(d)

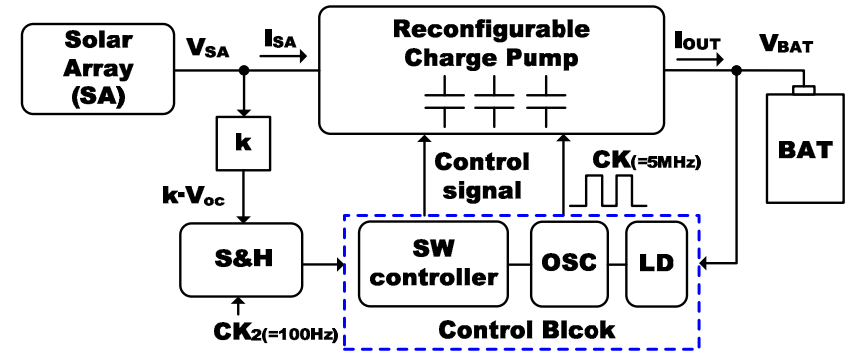

(e)

Fig. 4. (a) The circuit implementation of Figure 3 (a) of the proposed reconfigurable charge pump (RCP) circuit (b) the detailed single-stage charge pump circuit with three switches (c) the switch controller circuit (d) the $\mathrm{S} \& \mathrm{H}$ circuit (e) the entire block diagram of RCP circuit.

is on, the gate of $M_{S N}$ is driven by $V_{H}$ to turn off $M_{S N}$. When $M_{N}$ is on, the gate voltage of $\mathrm{M}_{\mathrm{SN}}$ is $0 \mathrm{~V}$ thus $\mathrm{M}_{\mathrm{SN}}$ becomes on. A simple level shifter is used to apply a high voltage, $\mathrm{V}_{\mathrm{H}}$, to $\mathrm{M}_{\mathrm{P}}$ [12]. $\mathrm{M}_{\mathrm{N}}$ can be controlled by the inverter, $\mathrm{I}_{0} . \Phi_{1}$ and $\Phi_{2}$ in Figure 4 (c) are the non-overlapping signals that are used to eliminate the direct current path that may happen when both $\mathrm{M}_{\mathrm{P}}$ and $\mathrm{M}_{\mathrm{N}}$ are on. The non-overlapping clocks are generated by the nonoverlapping clock generator that is popularly used [13]. In Figures 4 (b) and 
(c), only the non-overlapping circuit and I0 are made of normal devices. The others should be made of high-voltage devices to suffer high-voltage stress.

Figure $4(\mathrm{~d})$ shows the sample and hold $(\mathrm{S} \& \mathrm{H})$ circuit that can sample $\mathrm{k} \cdot \mathrm{V}_{\text {OC }}$ periodically to change the operation mode of charge pump [2]. The $\mathrm{S} \& \mathrm{H}$ circuit has two amplifiers, $\mathrm{A}_{1}$ and $\mathrm{A}_{2}$ that are the unit-gain buffer. The sampled voltage is stored at $C_{S}$. Two resistors, $R_{0}$ and $R_{1}$, can decide the fractional number $\mathrm{k}$ that is usually known between 0.7 and 0.8 in most of solar cells [1]. $\mathrm{S}_{\mathrm{C} 0}$ and $\mathrm{S}_{\mathrm{C} 1}$ are the control switches. $\mathrm{CK}_{2}$ and $\mathrm{CKB}_{2}$ are the sampling clocks that are very slow. In this paper, $\mathrm{CK}_{2}$ and $\mathrm{CKB}_{2}$ are decided around $100 \mathrm{~Hz}$ that is fast enough to track the MPP with sunlight variation. When $\mathrm{CK}_{2}$ is high, the sampled $\mathrm{k} \cdot \mathrm{V}_{\mathrm{OC}}$ is stored at $\mathrm{C}_{\mathrm{S}}$. When $\mathrm{CKB}_{2}$ is high, the stored $\mathrm{k} \cdot \mathrm{V}_{\mathrm{OC}}$ goes into the comparator to decide the operation mode in SM, SPM, and PM. One thing to note here is that we cannot use a continuous monitoring circuit to detect $\mathrm{k} \cdot \mathrm{V}_{\mathrm{OC}}$ in the RCP circuit. $\mathrm{V}_{\mathrm{OC}}$ means the open-circuit voltage that means the solar cell's current is zero. Thus the RCP circuit should stop its operation during the $\mathrm{k} \cdot \mathrm{V}_{\mathrm{OC}}$ sampling time when $\mathrm{CK}_{2}$ is high. In this paper, the sampling time when $\mathrm{CK}_{2}$ is high is as short as $10 \mu \mathrm{sec}$ thus the overall pumping speed is very little degraded. From the simulation, the battery charging time from $2.75 \mathrm{~V}$ to $4.2 \mathrm{~V}$ becomes slower only by less than $0.1 \%$ if the $\mathrm{k} \cdot \mathrm{V}_{\mathrm{OC}}$ sampling frequency $=100 \mathrm{~Hz}$ and $\mathrm{CK}_{2}$ high time $=10 \mu \mathrm{sec}$.

Figure 4 (e) shows the entire block diagram of RCP circuit. Here $\mathrm{V}_{\mathrm{SA}}$ and $\mathrm{I}_{\mathrm{SA}}$ are solar array voltage and current, respectively. If the sampled $\mathrm{k} \cdot \mathrm{V}_{\mathrm{OC}}$ is large, it means that the sunlight is very bright thus the charge pump can work by PM. If $\mathrm{k} \cdot \mathrm{V}_{\mathrm{OC}}$ is small, the pumping capacitors should be rearranged by SM. For moderate sunlight, the RCP changes to SPM. The fractional number, $\mathrm{k}$ is decided by $\mathrm{R}_{0}$ and $\mathrm{R}_{1}$ in Figure $4(\mathrm{~d})$. $\mathrm{S} \& \mathrm{H}$ circuit samples $\mathrm{k} \cdot \mathrm{V}_{\mathrm{OC}}$ with a very slow clock, $\mathrm{CK}_{2}$, as slow as $100 \mathrm{~Hz}$. The $\mathrm{SW}$ controller decides which mode is the most suitable to given sunlight intensity. This decision is made by the comparator circuits [14]. The SW controller has four comparators that generate 'DARK' mode, SM, SPM, and PM signals. The 'DARK' mode means that the sunlight is too weak to deliver enough power to the RCP circuit. For the 'DARK' mode, the SW controller turns off the clock oscillator (OSC) circuit thereby the RCP circuit stops its operation. After the decision, if not the 'DARK' mode, the SW controller gives either SM, SPM, or PM signal to the reconfigurable circuit to change the operation mode. $\mathrm{V}_{\mathrm{BAT}}$ is the battery voltage and $\mathrm{I}_{\mathrm{OUT}}$ is the output current of RCP circuit. $\mathrm{V}_{\mathrm{BAT}}$ is sensed by $\mathrm{LD}$ that is the $\mathrm{V}_{\mathrm{BAT}}$ level detector. If $\mathrm{V}_{\mathrm{BAT}}$ is lower than the target voltage, OSC turns on the pump clock signal to deliver $\mathrm{I}_{\mathrm{OUT}}$ to the battery. If $\mathrm{V}_{\mathrm{BAT}}$ is higher than the target voltage, OSC becomes off thereby the $\mathrm{V}_{\mathrm{BAT}}$ can be regulated not to exceed the target voltage.

For the start-up, if $\mathrm{k} \cdot \mathrm{V}_{\mathrm{OC}} / \mathrm{V}_{\text {OUT }}$ is lower than 0.2 at dark sunlight condition, the SW controller does not enable OSC, in Figure $4(\mathrm{e})$. If $\mathrm{k}$. $\mathrm{V}_{\text {OC }} / \mathrm{V}_{\text {OUT }}$ becomes higher than 0.2 , in other words, the sampled $\mathrm{k} \cdot \mathrm{V}_{\mathrm{OC}}$ become larger than $0.9 \mathrm{~V}$, OSC starts to generate the pumping clock. This start-up is reasonable because a very low $\mathrm{k} \cdot \mathrm{V}_{\mathrm{OC}}$ means the dark sunlight 
condition, where the solar cell cannot deliver enough voltage to current to the RCP circuit.

\section{Simulation and measurement}

Figure 5 (a) compares the output current between the fixed-mode circuits by $\mathrm{SM}, \mathrm{SPM}$, and PM, and the proposed reconfigurable charge pump (RCP) circuit. As mentioned in the section II, the proposed RCP can be changed among SM, SPM, or PM, according to the sampled $\mathrm{k} \cdot \mathrm{V}_{\mathrm{OC}}$. In the simulation, the switching frequency is fixed at $5 \mathrm{MHz}$. $\mathrm{V}_{\text {OUT }}$ is the output voltage of charge pump and is targeted by $4.2 \mathrm{~V}$. The SPICE parameters are obtained from $0.18-\mu \mathrm{m}$ TSMC CMOS process. When $\mathrm{k} \cdot \mathrm{V}_{\mathrm{OC}} / \mathrm{V}_{\text {OUT }}$ is smaller than 0.4 , in other words, when the sunlight is weak, the fixed SM circuit can deliver larger $\mathrm{I}_{\mathrm{OUT}}$ than the PM and SPM circuits. When $\mathrm{k} \cdot \mathrm{V}_{\mathrm{OC}} / \mathrm{V}_{\mathrm{OUT}}$ becomes between 0.4 and 0.6, the SPM circuit starts to deliver larger output current than the SM circuit, because the effective pumping capacitance of SPM is twice larger than the pumping capacitance of SM, as shown in Figure 3 (c). For strong sunlight, when $\mathrm{k} \cdot \mathrm{V}_{\mathrm{OC}} / \mathrm{V}_{\text {OUT }}$ is larger than 0.6 , the PM circuit can deliver the largest pumping current to the load among the SM, SPM, and $\mathrm{PM}$ circuit. For example, when $\mathrm{k} \cdot \mathrm{V}_{\mathrm{OC}} / \mathrm{V}_{\mathrm{OUT}}=0.8$, the amount of pumping current of the PM circuit reaches as large as $5 \mathrm{~mA}$ that is almost twice larger than the SPM circuit and four times larger than SM circuit in Figure 5 (a). In Figure 5 (a), the proposed reconfigurable circuit can follow the largest $\mathrm{I}_{\mathrm{OUT}}$ among the three PM, SPM, and SM circuits. For weak sunlight, the proposed RCP runs like the SM circuit. When the sunlight is moderate, the RCP shows the same amount of IOUT with the SPM circuit.

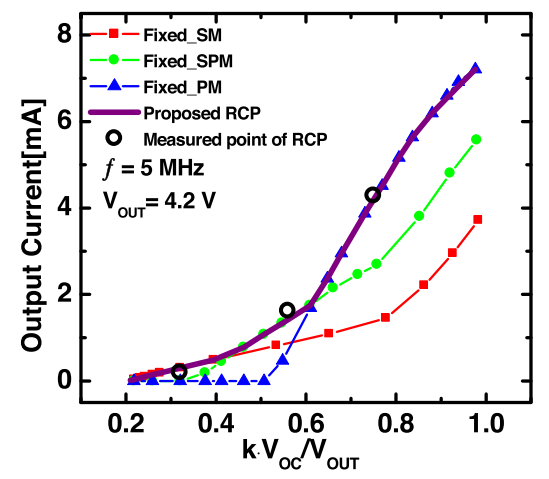

(a)

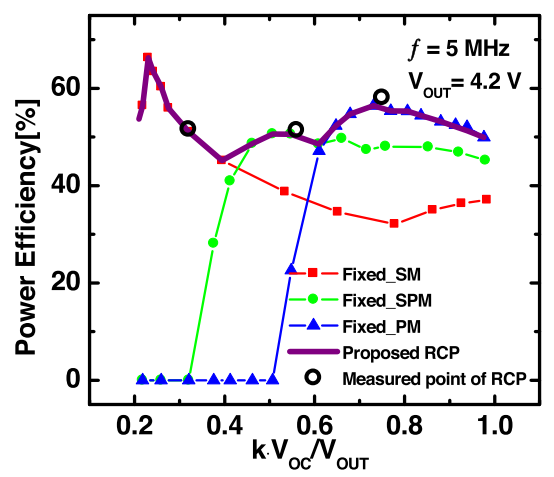

(b)

Fig. 5. (a) Output current with varying $\mathrm{k} \cdot \mathrm{V}_{\mathrm{OC}} / \mathrm{V}_{\text {OUT }}$ for the fixed SM circuit, the fixed SPM circuit, the fixed PM circuit, and the proposed RCP circuit. Here the open circle symbols are the measured results of the proposed RCP. (b) Power efficiency with varying $\mathrm{k} \cdot \mathrm{V}_{\mathrm{OC}} / \mathrm{V}_{\mathrm{OUT}}$ for the fixed SM circuit, the fixed SPM circuit, the fixed PM circuit, and the proposed RCP circuit. Here the open circle symbols are the measured results of the proposed RCP. 
For strong sunlight, the proposed circuit acts like the PM circuit to maximize $\mathrm{I}_{\mathrm{OUT}}$ to the load. From Figure 5 (a), we can obtain the boundary values of $\mathrm{k} \cdot \mathrm{V}_{\mathrm{OC}} / \mathrm{V}_{\text {OUT }}$ for the three modes. For the $\mathrm{SM}$ circuit, $\mathrm{k} \cdot \mathrm{V}_{\mathrm{OC}} / \mathrm{V}_{\text {OUT }}$ should be less than 0.4 to generate larger $\mathrm{I}_{\mathrm{OUT}}$ than the SPM and PM circuits. Here the boosting ratio can be defined by $\mathrm{V}_{\text {OUT }} / \mathrm{V}_{\mathrm{IN}}$. When $\mathrm{k} \cdot \mathrm{V}_{\mathrm{OC}} / \mathrm{V}_{\text {OUT }}=0.4$ i.e. $\mathrm{V}_{\text {OUT }} / \mathrm{k} \cdot \mathrm{V}_{\mathrm{OC}}=2.5$, the boosting ratio should be larger than 3 to generate $\mathrm{V}_{\text {OUT }}=4.2 \mathrm{~V}$. Among the SM, SPM, and PM circuits, only the SM can have the boosting ratio that is larger than 3 . When $\mathrm{k} \cdot \mathrm{V}_{\mathrm{OC}} / \mathrm{V}_{\text {OUT }}$ is between 0.4 and 0.6, the SPM circuit shows the largest $\mathrm{I}_{\mathrm{OUT}}$. Here the boosting ratio as large as 3 is enough to generate $\mathrm{V}_{\text {OUT }}=4.2 \mathrm{~V}$. When $\mathrm{k} \cdot \mathrm{V}_{\mathrm{OC}} / \mathrm{V}_{\text {OUT }}$ is larger than 0.6, the PM circuit has the largest IOUT among the three. At this region of $\mathrm{k} \cdot \mathrm{V}_{\mathrm{OC}} / \mathrm{V}_{\mathrm{OUT}}$, the boosting ratio as large as 2 can satisfy the target $\mathrm{V}_{\text {OUT }}$.

Figure 5 (b) compares the power efficiency between the fixed-mode circuits and the proposed RCP. The three fixed-mode circuits are SM, SMP, and PM, respectively. The power efficiency is defined by a ratio of output power to input power. Here the output power is calculated by $\mathrm{I}_{\text {OUT }} \times \mathrm{V}_{\text {OUT }}$ and the input power is calculated by $\mathrm{I}_{\mathrm{SA}} \times \mathrm{V}_{\mathrm{SA}}$. When $\mathrm{k} \cdot \mathrm{V}_{\mathrm{OC}} / \mathrm{V}_{\mathrm{OUT}}$ is between 0.2 and 0.4, the SM circuit shows better efficiency than the SPM and PM. For the SPM circuit, the power efficiency is better than the other two circuits of $\mathrm{SM}$ and $\mathrm{PM}$ when $\mathrm{k} \cdot \mathrm{V}_{\mathrm{OC}} / \mathrm{V}_{\text {OUT }}$ is between 0.4 and 0.6 . When $\mathrm{k} \cdot \mathrm{V}_{\mathrm{OC}} / \mathrm{V}_{\text {OUT }}$ is larger than 0.6, the PM can show the best efficiency among the 3 fixedmode circuits. As $\mathrm{k} \cdot \mathrm{V}_{\mathrm{OC}} / \mathrm{V}_{\text {OUT }}$ becomes larger than 0.75 , the efficiency of PM circuit begins to be decreased like the other two fixed-mode circuits. Like Figure 5 (a), the proposed RCP follows the best performance among the three SM, SPM, and PM circuits. This is because the proposed circuit can change its architecture according to the sunlight variation. In Figure 5 (b), the RCP shows the power efficiency better by $23.6 \%$ on average than the SM circuit. Comparing with the PM and SPM circuits, the RCP is better by $23.8 \%$ and $28.8 \%$, respectively. In Figure 5 (b), the boundary values of SM, SPM, and PM for the proposed RCP are the same with Figure 5 (a).

The measured data are listed in Table I. The fabrication technology was TSMC 0.18- $\mu \mathrm{m}$ CMOS process that included high-voltage $0.35-\mu \mathrm{m}$ devices.

Table I. The measured data of the proposed RCP circuit. 
Here the proposed RCP was compared with the three fixed-mode circuits that are SM, SPM, and PM, respectively. From Table I, when the sunlight is darkness, both the fixed-mode circuits and proposed RCP circuit cannot run at all thus the output current and power efficiency are zero. For weak sunlight, only SM circuit can start to run and can deliver the output current as large as $0.2 \mathrm{~mA}$ with the power efficiency as large as $51.6 \%$. But until this time, the other two fixed-mode circuits of SPM and PM cannot generate the output voltage as large as $4.2 \mathrm{~V}$ thus the delivered power of SPM and PM is zero. When sunlight is weak, the proposed RCP runs like the SM circuit thereby the output current can be as large as $0.2 \mathrm{~mA}$ and the power efficiency becomes $51.6 \%$ like the SM circuit. Similarly, when the sunlight condition is moderate, the RCP acts like the SPM circuit, where the output current is $1.62 \mathrm{~mA}$ and the efficiency is as large as $51.4 \%$. When sun shines very brightly, the proposed RCP rearranges all the pumping capacitors in parallel to maximize the output current. By doing so, the RCP can achieve the output current as large as $4.29 \mathrm{~mA}$ with the power efficiency as much as $58.1 \%$. Figure 6 shows the layout of the proposed RCP circuit. For the area penalty, the added switches, S\&H, OSC, LD, and SW controller occupy only $15.8 \%$ of the total area. The S\&H, OSC, LD, and SW controller circuits are labeled in Figure 6.

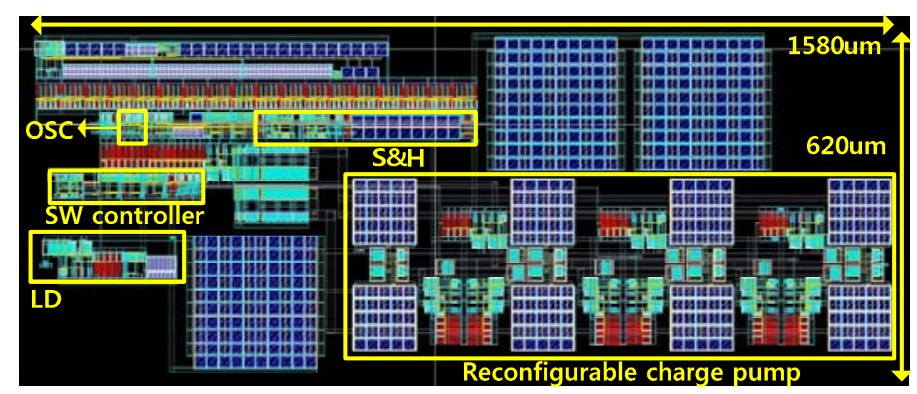

Fig. 6. The top view of layout with TSMC $0.18-\mu \mathrm{m}$ CMOS process.

One thing to note is that the three fixed-mode circuits of SM, SPM, and PM do not need the control switches, SW controller, and S\&H circuit because they do not reconfigure the pumping capacitors. Hence, to estimate the power overhead from the added blocks for reconfiguration, we compared the fixedmode circuits without any reconfiguration-related blocks to the RCP circuit. For weak sunlight, the difference in power efficiency between the pure charge pump circuit without reconfiguration and the proposed RCP circuit is as little as $1.2 \%$ that can almost be neglected. When the sunlight is moderate, the difference in efficiency becomes smaller to $0.4 \%$. If the sunlight becomes strong, the efficiency difference between the pure charge pump and the RCP circuit is only $0.13 \%$. The one big reason that the power overhead due to the reconfiguration is so small is that the $\mathrm{k} \cdot \mathrm{V}_{\mathrm{OC}}$ sampling frequency as slow as $100 \mathrm{~Hz}$ is too much slow to affect the total power consumption that is mostly dominated by the pumping operation run by $5 \mathrm{MHz}$. 


\section{Conclusion}

In this paper, we proposed the reconfigurable charge pump (RCP) that could change its architecture according to sunlight-intensity variation. In the proposed RCP, when the sunlight is strong, the charge pump changes its architecture to Parallel Mode (PM), where 4 pumping capacitors are connected in parallel to deliver the maximum output current to the load. When the sunlight becomes moderate, the circuit rearranges its pumping capacitors by Serial-Parallel Mode (SPM), where the output current and power efficiency are traded off each other to give the best performance at this sunlight condition. In weak sunlight, the RCP acts as Serial Mode (SM), where all the pumping capacitors are in series to increase the boosting ratio higher.

From the measured data, the proposed RCP can deliver 2.5 times and 1.4 times larger output current than the SM and SPM circuits, respectively, in strong sunlight. For the power efficiency, the RCP is better by $24 \%$ and $7 \%$ than the SM and SPM circuit, respectively.

\section{Acknowledgments}

This work was financially supported by the SRC/ERC program (R11-2005048-00000-0), the Basic Science Research Program (2011-0026132), the Global Research Network Program (NRF-2011-220-D00089), the NanoMaterial Technology Development Program (2011-0030228) through the National Research Foundation of Korea (NRF) funded by the Ministry of Education, Science and Technology, and the Industrial Strategic Technology Development Program funded by the Ministry of Knowledge Economy (MKE, Korea) (10039239). The CAD tools were supported by the IC Design Education Center (IDEC), Korea. 